(c) Eurographics Association, 1993

\title{
Variational Surface Design and Surface Interrogation
}

\author{
H. Hagen, St. Hahmann \\ Universität Kaiserslautern \\ FB Informatik \\ Postfach 3049 \\ D-6750 Kaiserslautern
}

\author{
G.-P. Bonneau \\ CEA \\ Centre de Recherches de Limeil-Valenton \\ F-94195 Villeneuve St. Georges Cedex
}

\begin{abstract}
The generation of technical smooth surfaces from a mesh of three-dimensional data points is an important problem in geometric modelling. In this publication we give a survey of some new techniques based on a calculus of variation approach. Apart from the pure construction of these surfaces, the analysis of their quality is equally important in the design and manufacturing process. Generalized focal surfaces are presented here as a new surface interrogation tool.
\end{abstract}

\section{o Introduction}

Curves and surfaces designed in a Computer Graphics environment have many applications, including the design of cars, air planes, shipbodies and modeling robots. The generation of "technical smooth" surfaces which are appropriate for the NC-process from a set of threedimensional data points is a key problem in the field of Computer Aided GeometricDesign. The fundamental idea for the described methods is the use of modeling tools which minimize a certain functional that can be interpretated in the sense of physics and/or geometry. In chapter 2 we deal with a variational design method for B-Spline-Surfaces. This concept is extended to NURBS-surfaces in chapter 3.

In chapter 4 we present generalized focal surfaces as a new tool for surface interrogation.

\section{Fundamentals}

We keep this chapter short, because we assume that reader is familiar with the basic concepts of the Bezier and B-Spline techniques.

The curves now known as Bezier curves and surfaces were independently developed by P. de Casteljau and by P. Bezier. The underlying mathematical theory, based on the concept of Bernstein polynomials, was first introduced by R. Forrest (see [For72]). The fundamental idea of this approach is to evaluate and manipulate the curves by a (small) number of "control 
points". A Bezier-curve is a segmented curve. The segments $X_{\ell}(u), \ell=0, \cdots, k$ of a Beziercurve of degree $m$ over the parameter interval $u_{\ell} \leq u \leq u_{\ell+1}$ are:

$$
X_{\ell}(u):=\sum_{i=0}^{m} b_{\ell+i} \cdot B_{i}^{m}\left(\frac{u-u_{\ell}}{u_{\ell+1}-u_{\ell}}\right)
$$

The Bernstein polynomials $B_{i}^{m}(t):=\left(\begin{array}{c}m \\ i\end{array}\right)(1-t)^{m-i} t^{i} ; 0 \leq t \leq 1$ are used as blending functions. This concept can be extended to NURBS (non-uniform rational B-splines). The fundamental idea of the rational Bezier- and B-spline algorithms is to evaluate and manipulate the curves and surfaces by a (small) number of control points and so called weights. These weights are additional design parameters. First we give the fundamental concepts of rational curves, following [Far90]. A rational Bezier curve of degree $n$ in $\mathbf{E}^{3}$ is the projection of an $n$th degree Bezier curve in $\mathbf{E}^{4}$ onto the hyperplane $w=1$.

$$
x(t)=\frac{w_{0} \mathbf{b}_{0} \mathbf{B}_{0}^{n}(t)+\cdots+w_{n} \mathbf{b}_{n} \mathbf{B}_{n}^{n}(t)}{w_{0} B_{0}^{n}(t)+\cdots+w_{n} B_{n}^{n}(t)} .
$$

$x(t), \mathbf{b}_{i} \in \mathbf{E}^{3} ; w_{i} \geq 0$ are called (scalar) weights.

We reparametrize a rational Bezier curve by changing the weights according to

$$
\widetilde{w}_{i} ;=c^{\prime} w_{i} ; \quad i=0, \ldots, n ; \quad c:=\left(w_{0} / w_{n}\right)^{1 / n}
$$

(see [Far90]) and get, after dividing all weights by $w_{0}$, the standard form

$$
x(t)=\frac{\sum_{i=0}^{n} w, \mathbf{b}_{i} \mathbf{B}_{i}^{n}(t)}{\sum_{i=0}^{n} w_{i} \mathbf{B}_{i}^{n}(t)}: w_{0}=w_{n}=1 ; \quad w_{i}>0
$$

This yields in the rational cubic case:

$$
x(t)=\frac{\mathbf{b}_{0} \mathbf{B}_{0}^{3}(t)+w_{\ell} \mathbf{b}_{\ell} \mathbf{B}_{\ell}^{3}(t)+w_{2} \mathbf{b}_{2} \mathbf{B}_{2}^{3}(t)+\mathbf{b}_{3}^{3} \mathbf{B}_{3}^{3}(t)}{\mathbf{B}_{0}^{3}(t)+w_{\ell} \mathbf{B}_{\ell}^{3}(t)+w_{2} \mathbf{B}_{2}^{3}(t)+\mathbf{B}_{3}^{3}(t)}
$$

A Bezier-surface is a segmented surface. The segments $X_{p q}(u, w): P=0, \ldots, r$ of a Bezier surface of degree $m, n$ over the rectangular parameter domain $u_{p} \leq u \leq u_{p+1}: w_{q} \leq w \leq w_{q+1}$ are

$$
X_{p q}(u, w):=\sum_{i=0}^{m} \sum_{j=0}^{n} b_{p \cdot m+i / q \cdot n+j} \cdot B_{i}^{m}\left(\frac{u-u_{p}}{u_{p+1}-u_{p}}\right) B_{j}^{n}\left(\frac{w-w_{q}}{w_{q+1}-w_{q}}\right)
$$

Instead of a control polygon a Bezier-surface(-segment) has a control polyhedron.

The definition of a B-spline-surface over a rectangular parameter domain follows directly the same pattern:

$$
Y(u, w):=\sum_{i=0}^{m} \sum_{j=0}^{n} d_{i j} N_{i, M}(u) N_{j, N}(w)
$$

A rational Bezier-surface $X(u, v)$ is definded by

$$
X(u, v)=\frac{\sum_{i=0}^{n} \sum_{j=0}^{m} w_{i j} b_{i j} B_{i}^{n}(u) B_{j}^{m}(v)}{\sum_{i=0}^{n} \sum_{j=0}^{m} w_{i j} B_{i}^{n}(u) B_{j}^{m}(v)}, w_{i j}>0
$$

as the projection of a $4 \mathrm{D}$-tensor product Bezier-surface. But it is common misconception to call (8) a tensor product surface itself.

For more details about these basic concepts see [Far90] and [HL89]. 


\section{Variational design of B-spline surfaces}

In this section we leave the classical approach

- construct a smooth net of curves

- add the surface patches smoothly into the net

and present a direct method to construct a technically smooth B-Spline spline surface, which uses only point data and refrains from determining a net.

The construction algorithm combines a weighted least square approximation with automatic surface smoothing. The smoothing criterion is the approximate minimization of the curvature variation. This technique presented here aims at constructing tangent-plane continuous Bspline surfaces. The following mathematical model serves as variation principle:

$$
\begin{aligned}
(1-w s) & \left\{\sum_{k=1}^{n_{p}} w_{p k}\left[X\left(u_{k}, v_{k}\right)-P_{k}\right]^{2}\right\} \\
+ \text { ws } \quad & \left\{\sum_{i=1}^{n} \sum_{j=1}^{m} w_{3_{u}} \int_{v_{j}}^{v_{j+1}} \int_{u_{i}}^{u_{i+1}} w_{3_{u i j}}\left\|\frac{\partial^{3} X(u, v)}{\partial u^{3}}\right\|^{2} d u d v\right. \\
+ & \left.w_{3_{v}} \int_{v_{j}}^{v_{j+1}} \int_{u_{i}}^{u_{i+1}} w_{3_{i j}}\left\|\frac{\partial^{3} X(u, v)}{\partial v^{3}}\right\|^{2} d u d v\right\} \rightarrow \min .
\end{aligned}
$$

$X(u, \mathrm{v})$ is the representation of the surface, $(u, v) \in\left[u_{1}, u_{n+1}\right] \times\left[v_{1}, v_{m+1}\right]$ is the parameter value and $n, m$ are the number of segments in $u$ - and v-direction. $P_{k}$ are the points to be approximated and $n_{p}$ is the number of these points. The weight coefficients $w s, w_{3 u}, w_{3 v}, w_{3} u_{i j} w_{3} v_{i j}$ are valid in the interval $[0,1]$ and fulfill the constraints $\sum_{i=1}^{m} \sum_{j=1}^{n} w_{3 u_{i j}}=1$ and $\sum_{i=1}^{m} \sum_{j=1}^{n} w_{3 v_{j i}}^{r}=$ 1. We apply this variation principle tu biquintic b-spline surfaces

$$
X(u, v)=\sum_{i=1}^{4 n+2} \sum_{j=1}^{4 m+2} d_{i j} N_{i}^{5}(u) N_{j}^{5}(v)
$$

with the knot-vectors

$$
\left\{k_{1}^{u}, \ldots, k_{4 n+8}^{u}\right\}:=\{\underbrace{u_{1}, \ldots, u_{1}}_{6 m}, \underbrace{u_{2}, u_{2}, u_{2}, u_{2}}_{4 m}, \cdots, \underbrace{u_{n}, u_{n}, u_{n}, u_{n}}_{4 m}, \underbrace{u_{n+1}, \ldots, u_{n+1}}_{6 n}\}
$$

and

$$
\left\{k_{1}^{v}, \ldots, k_{4 m+8}^{v}\right\} ;=\{\underbrace{v_{1}, \ldots, v_{1}}_{6 m}, \underbrace{v_{2}, v_{2}, v_{2}, v_{2}}_{4 m}, \cdots, \underbrace{v_{m}, v_{m} v_{m} v_{m}}_{4 m}, \underbrace{v_{m+1}, \ldots, v_{m+1}}_{6 n}\} .
$$

This set of knot vectors guarantees the $C^{1}$-continuity of the surface. We can now use the control points $d_{i j}(i \in\{1, \ldots ; 4 n+2\}, j \in\{1, \ldots, 4 m+2\})$ as parameters for the calculus of variation approach. Applying the variation principle (9) is a three step process. 
Step 1. Least square fitting.

$$
S L:=\sum_{k=1}^{n_{p}} w_{p k}\left[F\left(u_{k}, v_{k}\right)-\left.P_{k}\right|^{2} \rightarrow \min .\right.
$$

or in B-spline representation

$$
\sum_{k=1}^{n_{p}} w_{p k}\left[\sum_{\ell=1}^{4 n+2} \sum_{r=1}^{4 m+2} d_{\ell r} N_{\ell}^{5}\left(u_{k}\right) N_{r}^{5}\left(v_{k}\right)-P_{k}\right]^{2} \rightarrow \min .
$$

The necessary conditions $\frac{\partial S L}{\partial d_{i j}}=0$ lead to a linear system of equations:

$$
\sum_{\ell=1}^{4 n+2} \sum_{r=1}^{4 m+2}\left\{2 \sum_{k=1}^{n_{p}} w_{p k} \dot{N}_{\ell}^{5}\left(u_{k}\right) N_{r}^{5}\left(v_{k}\right) N_{i}^{5}\left(u_{k}\right) N_{j}^{5}\left(v_{k}\right)\right\} d_{\ell r}=2 \sum_{k=1}^{n_{p}} w_{p k} \dot{P}_{k} N_{i}^{5}\left(u_{k}\right) N_{j}^{5}\left(v_{k}\right)
$$

The unique solution of this system is the best point fitting in the least square sense of (11). Step 2. Automated smoothing process:

As a fairness criterion we use

$$
\sum_{i=1}^{n} \sum_{j=1}^{m} \int_{v_{j}}^{v_{j}+1} w 3 u \dot{w} 3 u_{i j}\left|\frac{\partial^{3} X(u, v)}{\partial^{3}}\right|^{2}+w 3 v \cdot w 3 v_{i j}\left|\frac{\partial^{3} X(u, v)}{\partial^{3}}\right|^{2} d u d v \rightarrow \text { min. }
$$

A calculus of variation approach leads to a linear system of equations, which can be found in detail in [HS91].

\section{Step 3.}

We now conbine the weighted least square fit with the automated smoothing process

$$
(1-w s) A+w s B=0
$$

A symbolizes equations (13) and $B$ symbolizes the equations of the 2. step.

Applications We use this method to construct reflection surfaces for car headlights. 
Step 1. Digitizing

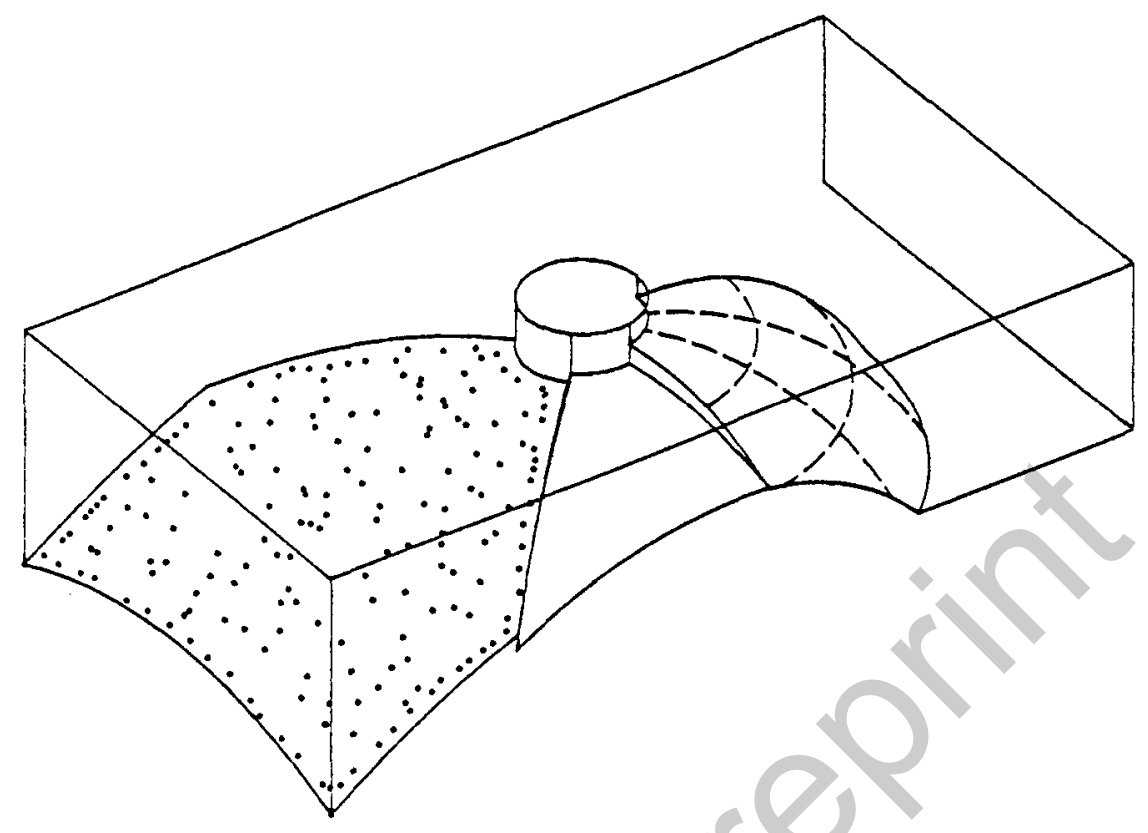

Figure 1:

Step 2. Parametrization

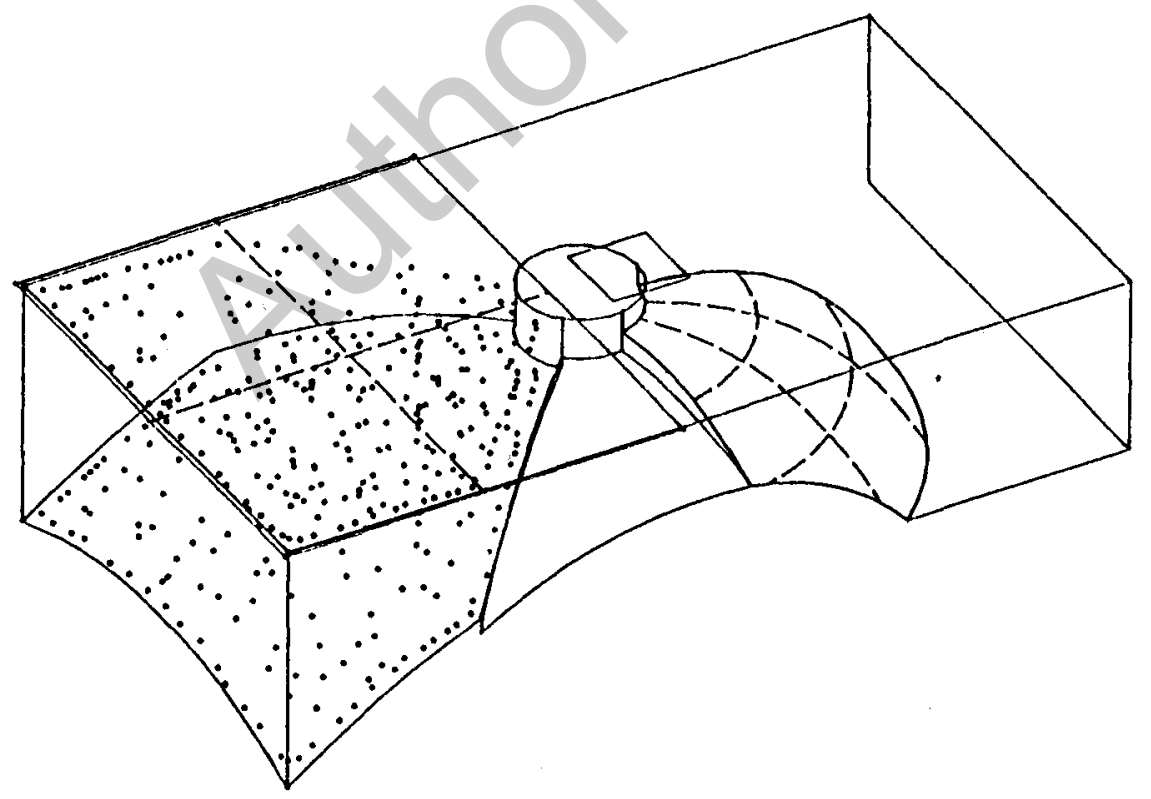

Figure 2: 
Step 3. Variational Surface Design

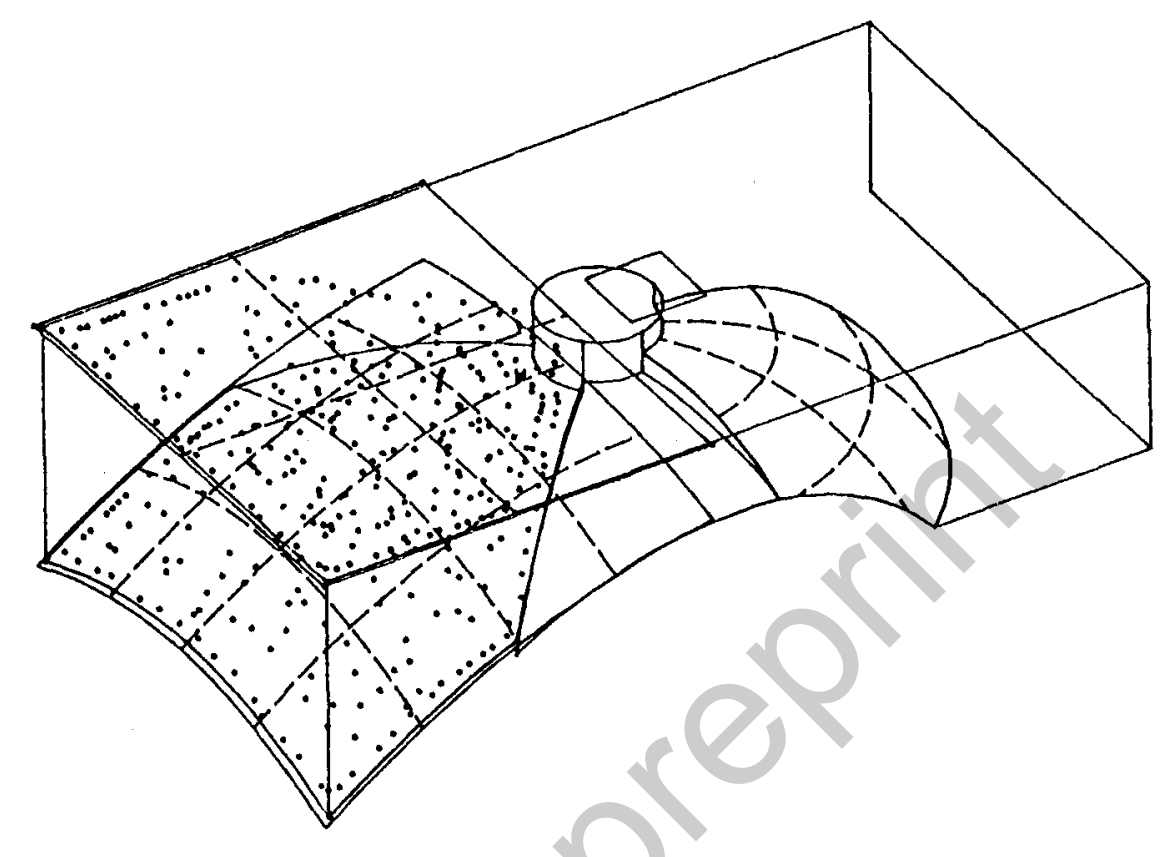

Figure 3: 


\section{Variational Design of Smooth Rational Bezier-Surfaces}

The purpose of this chapter is to present an algorithm to assign to the weights appropriate values to achieve technical smooth surfaces. The standard fairness criterion for surfaces in engineering is to minimize the strain energy of flexure and torsion in a thin rectangular elastic plate of small deflection. The functional $\int_{S}\left(\kappa_{1}^{2}+\kappa_{2}^{2}\right) d s\left(\kappa_{1}\right.$ and $\kappa_{2}$ are the principal curvatures of the surface $S$ ) is a mathematical model for the energy stored in such a plate. We approximate this integral criterion by a quadrature formula. A calculus of variation approach based upon the integral criterion with respect to the weights of the rational surfaces, leads to a linear (!) system of equations. The unique solution of this system gives appropriate weights for smooth surface design.[HB93]

A rational Bezier curve of degree $\mathrm{n}$ in $\mathbf{E}^{3}$ is the projection of an $\mathrm{nth}$ degree Bezier curve in $\mathbf{e}^{4}$ onto the hyperplane $w=1$.

$$
X(t)=\frac{w_{0} b_{0} B_{0}^{n}(t)+\cdots+w_{n} b_{n} B_{n}^{n}(t)}{w_{0} B_{0}^{n}(t)+\cdots w_{n} B_{n}^{n}(t)}
$$

$X(t), b_{i} \in E^{3} ; w_{i}>0$ are called (scalar) weights. A rational Bezier-surface $X(u, v)$ is definded by

$$
X(u, v)=\frac{\sum_{i=0}^{n} \sum_{j=0}^{m} w_{i j} b_{i j} B_{i}^{n}(u) B_{j}^{m}(v)}{\sum_{i=0}^{n} \sum_{j=0}^{m} w_{i j} B_{i}^{n}(u) B_{j}^{m}(v)}, w_{i} j>0
$$

as the projection of a 4D-tensor product Bezier-surface. But it is common misconception to call (16) a tensor product surface itself.

In the case of surfaces a thin elastic plate of small deflection can serve as a model for a fair shape. Such a plate tends to take a position of least strain energy of flexure and torsion. The energy stored in this plate is proportional to the integral

$$
G:=\int_{S}\left(\kappa_{1}^{2}+\kappa_{2}^{2}\right) d s
$$

$\kappa_{1}$ and $\kappa_{2}$ are the principle curvatures of the surface $S$. In our NURBS-setting:

$$
\begin{aligned}
\int_{S}\left(\kappa_{1}^{2}+\kappa_{2}^{2}\right) d s & =\int_{u_{1}}^{u_{2}} \int_{v_{1}}^{u_{2}} \frac{\left(g_{11} h_{22}-2 g_{12} h_{12}+g_{22} h_{11}\right)^{1}-2 g h}{g^{2}} \sqrt{ } g d u d v \\
h_{11} & :=<X_{u u}, N>\quad g_{11}:=<X_{U}, X_{U}> \\
h_{22} & :=<X_{v v}, N>\quad g_{22}:=<X_{v}, X_{v}> \\
g & :=g_{11} g_{22}-g_{12}^{2} \\
h & :=h_{11} h_{22}-h_{12}^{2} \\
N & :=\frac{\left[X_{u}, X_{v}\right]}{\left.\| X_{u}, X_{v}\right] \|}
\end{aligned}
$$

As an approcimation of this integral we use

$$
\int_{u_{1}}^{u_{2}} \int_{v_{1}}^{v_{2}} f(u, v) d u d v \simeq \frac{\left(u_{2}-u_{1}\right)\left(v_{2}-v_{1}\right)}{4}\left(f\left(u_{1}, v_{1}\right)+f\left(u_{2}, v_{1}\right)+f\left(u_{1}, v_{2}\right)+f\left(u_{2}, v_{2}\right)\right)
$$

Using approximation (19) for a rational bicubic Bezier-surface, we get 
$\int_{S}\left(\kappa_{1}^{2}+\kappa_{2}^{2}\right) d s \simeq$

\section{1}

$2 \omega_{10}^{3} \omega_{01}^{3}\left(<b_{10}-b_{00}, b_{10}-b_{00}><b_{01}-b_{00}, b_{01}-b_{00}>-\left\langle b_{10}-b_{00}, b_{01}-b_{00}\right\rangle^{2}\right)^{\frac{3}{2}}$

$\times\left[2 \omega_{10}^{4} \omega_{02}^{2}<b_{10}-b_{00}, b_{10}-b_{00}>^{2}<b_{02}-b_{00}, N_{00}>^{2}\right.$

$+9 \omega_{10}^{2} \omega_{01}^{2} \omega_{11}^{2}<b_{10}-b_{00}, b_{01}-b_{00}>^{2}<b_{11}-b_{00}, N_{00}>^{2}$

$+2 \omega_{01}^{4} \omega_{20}^{2}<b_{01}-b_{00}, b_{01}-b_{00}>^{2}<b_{20}-b_{00}, N_{00}>^{2}$

$\left.-12 \omega_{10}^{3} \omega_{01} \omega_{11} \omega_{02}<b_{10}-b_{00}, b_{10}-b_{00}\right\rangle\left\langle b_{10}-b_{00}, b_{01}-b_{00}\right\rangle\left\langle b_{11}-b_{00}, N_{00}\right\rangle\left\langle b_{02}-b_{00}, N_{00}\right\rangle$

$\left.-12 \omega_{10} \omega_{01}^{3} \omega_{20} \omega_{11}<b_{10}-b_{00}, b_{01}-b_{00}\right\rangle\left\langle b_{01}-b_{00}, b_{01}-b_{00}\right\rangle\left\langle b_{20}-b_{00}, N_{00}\right\rangle\left\langle b_{11}-b_{00}, N_{00}>\right.$ $\left.+9 \omega_{10}^{2} \omega_{01}^{2} \omega_{11}^{2}<b_{10}-b_{00}, b_{10}-b_{00}\right\rangle\left\langle b_{01}-b_{00}, b_{01}-b_{00}\right\rangle\left\langle b_{11}-b_{00}, N_{00}\right\rangle^{2}$

$\left.\left.\left.+4 \omega_{10}^{2} \omega_{01}^{2} \omega_{20} \omega_{02}<b_{10}-b_{00}, b_{01}-b_{00}\right\rangle^{2}<b_{20}-b_{00}, N_{00}\right\rangle\left\langle b_{02}-b_{00}, N_{00}\right\rangle\right]$

1

$\overline{2 \omega_{20}^{3} \omega_{31}^{3}\left(<b_{20}-b_{03}, b_{20}-b_{03}><b_{31}-b_{03}, b_{31}-b_{03}>-<b_{13}-b_{03}, b_{02}-b_{03}>^{2}\right)^{\frac{3}{2}}}$

$\times\left[2 \omega_{20}^{4} \omega_{32}^{2}<b_{20}-b_{03}, b_{20}-b_{03}>^{2}<b_{32}-b_{03}, N_{03}>^{2}\right.$

$+9 \omega_{20}^{2} \omega_{31}^{2} \omega_{21}^{2}<b_{13}-b_{03}, b_{02}-b_{03}>^{2}<b_{21}-b_{03}, N_{03}>$

$+2 \omega_{31}^{4} \omega_{10}^{2}<b_{31}-b_{03}, b_{31}-b_{03}>^{2}<b_{10}-b_{03}, N_{03}>^{2}$

$\left.\left.-12 \omega_{20}^{3} \omega_{31} \omega_{21} \omega_{32}<b_{20}-b_{03}, b_{20}-b_{03}\right\rangle<b_{13}-b_{03}, b_{02}-b_{03}><b_{21}-b_{03}, N_{03}\right\rangle<b_{32}-b_{03}, N_{03}>$

$\left.\left.-12 \omega_{20} \omega_{31}^{3} \omega_{10} \omega_{21}<b_{13}-b_{03}, b_{02}-b_{03}\right\rangle\left\langle b_{31}-b_{03}, b_{31}-b_{03}\right\rangle\left\langle b_{10}-b_{03}, N_{03}\right\rangle<b_{21}-b_{03}, N_{03}\right\rangle$

$\left.+9 \omega_{20}^{2} \omega_{31}^{2} \omega_{21}^{2}<b_{20}-b_{03}, b_{20}-b_{03}\right\rangle\left\langle b_{31}-b_{03}, b_{31}-b_{03}\right\rangle\left\langle b_{21}-b_{03}, N_{03}\right\rangle^{2}$

$\left.\left.\left.+4 \omega_{20}^{2} \omega_{31}^{2} \omega_{10} \omega_{32}<b_{13}-b_{03}, b_{02}-b_{03}\right\rangle^{2}<b_{10}-b_{03}, N_{03}\right\rangle\left\langle b_{32}-b_{03}, N_{03}\right\rangle\right]$

\section{1}

$2 \omega_{13}^{3} \omega_{02}^{3}\left(<b_{13}-b_{30}, b_{13}-b_{30}><b_{02}-b_{30}, b_{02}-b_{30}>-<b_{20}-b_{30}, b_{31}-b_{30}>^{2}\right)^{\frac{3}{2}}$

$\times\left[2 \omega_{13}^{4} \omega_{01}^{2}<b_{13}-b_{30}, b_{13}-b_{30}>^{2}<b_{01}-b_{30}, N_{30}>^{2}\right.$

$+9 \omega_{13}^{2} \omega_{02}^{2} \omega_{12}^{2}<b_{20}-b_{30}, b_{31}-b_{30}>^{2}<b_{12}-b_{30}, N_{30}>^{2}$

$+2 \omega_{02}^{4} \omega_{23}^{2}<b_{02}-b_{30}, b_{02}-b_{30}>^{2}<b_{23}-b_{30}, N_{30}>^{2}$

$-12 \omega_{13}^{3} \omega_{02} \omega_{12} \omega_{01}\left\langle b_{13}-b_{30}, b_{13}-b_{30}\right\rangle\left\langle b_{20}-b_{30}, b_{31}-b_{30}\right\rangle\left\langle b_{12}-b_{30}, N_{30}\right\rangle\left\langle b_{01}-b_{30}, N_{30}\right\rangle$

$\left.-12 \omega_{13} \omega_{02}^{3} \omega_{23} \omega_{12}<b_{20}-b_{30}, b_{31}-b_{30}\right\rangle\left\langle b_{02}-b_{30}, b_{02}-b_{30}><b_{23}-b_{30}, N_{30}><b_{12}-b_{30}, N_{30}>\right.$

$+9 \omega_{13}^{2} \omega_{02}^{2} \omega_{12}^{2}\left\langle b_{13}-b_{30}, b_{13}-b_{30}\right\rangle\left\langle b_{02}-b_{30}, b_{02}-b_{30}\right\rangle\left\langle b_{12}-b_{30}, N_{30}\right\rangle^{2}$

$\left.+4 \omega_{13}^{2} \omega_{02}^{2} \omega_{23} \omega_{01}<b_{20}-b_{30}, b_{31}-b_{30}>^{2}<b_{23}-b_{30}, N_{30}><b_{01}-b_{30}, N_{30}>\right]$

$$
+\frac{1}{2 \omega_{23}^{3} \omega_{32}^{3}\left(<b_{23}-b_{33}, b_{23}-b_{33}><b_{32}-b_{33}, b_{32}-b_{33}>-<b_{32}-b_{33}, b_{23}-b_{33}>^{2}\right)^{\frac{3}{2}}} \times
$$




$$
\begin{aligned}
& \times\left[2 \omega_{23}^{4} \omega_{31}^{2}<b_{23}-b_{33}, b_{23}-b_{33}>^{2}<b_{31}-b_{33}, N_{33}>^{2}\right. \\
& +9 \omega_{23}^{2} \omega_{32}^{2} \omega_{22}^{2}<b_{32}-b_{33}, b_{23}-b_{33}>^{2}<b_{22}-b_{33}, N_{33}>^{2} \\
& +2 \omega_{32}^{4} \omega_{13}^{2}<b_{32}-b_{33}, b_{32}-b_{33}>^{2}<b_{13}-b_{33}, N_{33}>^{2} \\
& -12 \omega_{23}^{3} \omega_{32} \omega_{22} \omega_{31}<b_{23}-b_{33}, b_{23}-b_{33}><b_{32}-b_{33}, b_{23}-b_{33}><b_{22}-b_{33}, N_{33}><b_{31}-b_{33}, N_{33}> \\
& -12 \omega_{23} \omega_{32}^{3} \omega_{13} \omega_{22}<b_{32}-b_{33}, b_{23}-b_{33}><b_{32}-b_{33}, b_{32}-b_{33}><b_{13}-b_{33}, N_{33}><b_{22}-b_{33}, N_{33}> \\
& +9 \omega_{23}^{2} \omega_{32}^{2} \omega_{22}^{2}<b_{23}-b_{33}, b_{23}-b_{33}><b_{32}-b_{33}, b_{32}-b_{33}><b_{22}-b_{33}, N_{33}>^{2} \\
& \left.+4 \omega_{23}^{2} \omega_{32}^{2} \omega_{13} \omega_{31}<b_{32}-b_{33}, b_{23}-b_{33}>^{2}<b_{13}-b_{33}, N_{33}><b_{31}-b_{33}, N_{33}>\right]
\end{aligned}
$$

This criterion is now used for a calculus of variation approach, with $\omega_{11}, \omega_{12}, \omega_{21}, \omega_{22}$, as variation parameters. This leads to a linear system of equations! Assuming that none of the triples $\left\{b_{10}-b_{00}, b_{01}-b_{00}, b_{11}-b_{00}\right\},\left\{b_{20}-b_{30}, b_{31}-b_{30}, b_{21}-b_{30}\right\},\left\{b_{02}-b_{03}, b_{13}-b_{03}, b_{12}-\right.$ $\left.b_{03}\right\},\left\{b_{23}-b_{33}, b_{32}-b_{33}, b_{22}-b_{33}\right\}$ are coplanar, this linear system has the unique solution:

$$
\begin{aligned}
& \omega_{11}=\frac{\omega_{10}^{2} \omega_{20}<b_{10}-b_{00}, b_{10}-b_{00}><b_{20}-b_{00}, N_{00}>+\omega_{01}^{2} \omega_{02}<b_{01}-b_{00}, b_{01}-b_{00}><b_{02}-b_{00}, N_{00}>}{\left.\omega_{10} \omega_{01}\left(<b_{10}-b_{00}, b_{10}-b_{00}><b_{01}-b_{00}, b_{01}-b_{00}>+<b_{10}-b_{00}, b_{01}-b_{00}\right\rangle^{2}\right)} \\
& \times \frac{\left\langle b_{10}-b_{00}, b_{01}-b_{00}\right\rangle}{\left\langle b_{11}-b_{00}, N_{00}\right\rangle} \\
& \omega_{12}=\frac{\left.\left.\left.\omega_{13}^{2} \omega_{23}<b_{13}-b_{03}, b_{13}-b_{03}\right\rangle<b_{23}-b_{03}, N_{03}>+\omega_{02}^{2} \omega_{01}<b_{02}-b_{03}, b_{02}-b_{03}\right\rangle<b_{01}-b_{03}, N_{03}\right\rangle}{\left.\omega_{13} \omega_{02}\left(<b_{13}-b_{03}, b_{13}-b_{03}><b_{02}-b_{03}, b_{02}-b_{03}>+<b_{13}-b_{03}, b_{02}-b_{03}\right\rangle^{2}\right)} \\
& \times \frac{\left\langle b_{13}-b_{03}, b_{02}-b_{03}>\right.}{<b_{12}-b_{03}, N_{03}>} \\
& \omega_{21}=\frac{\omega_{20}^{2} \omega_{10}<b_{20}-b_{30}, b_{20}-b_{30}><b_{10}-b_{30}, N_{30}>+\omega_{31}^{2} \omega_{32}<b_{31}-b_{30}, b_{31}-b_{30}><b_{32}-b_{30}, N_{30}>}{\left.\omega_{20} \omega_{31}\left(<b_{20}-b_{30}, b_{20}-b_{30}><b_{31}-b_{30}, b_{31}-b_{30}>+<b_{20}-b_{30}, b_{31}-b_{30}\right\rangle^{2}\right)} \\
& \times \frac{\left\langle b_{20}-b_{30}, b_{31}-b_{30}>\right.}{<b_{21}-b_{30}, N_{30}>} \\
& \omega_{22}=\frac{\omega_{23}^{2} \omega_{13}<b_{23}-b_{33}, b_{23}-b_{33}><b_{13}-b_{33}, N_{33}>+\omega_{32}^{2} \omega_{31}<b_{32}-b_{33}, b_{32}-b_{33}><b_{31}-b_{33}, N_{33}>}{\left.\omega_{23} \omega_{32}\left(<b_{23}-b_{33}, b_{23}-b_{33}><b_{32}-b_{33}, b_{32}-b_{33}>+<b_{32}-b_{33}, b_{23}-b_{33}\right\rangle^{2}\right)} \\
& \times \frac{\left\langle b_{32}-b_{33}, b_{23}-b_{33}\right\rangle}{\left\langle b_{22}-b_{33}, N_{33}\right\rangle}
\end{aligned}
$$

This weight coefficients are considered optimal in the sense $\int_{S}\left(\kappa_{1}^{2}+\kappa_{2}^{2}\right) d s \rightarrow \min$. 


\section{Applications}

The next picture shows a bicubic rational Bezier-surface with weights equal to one and with our optimal weights.

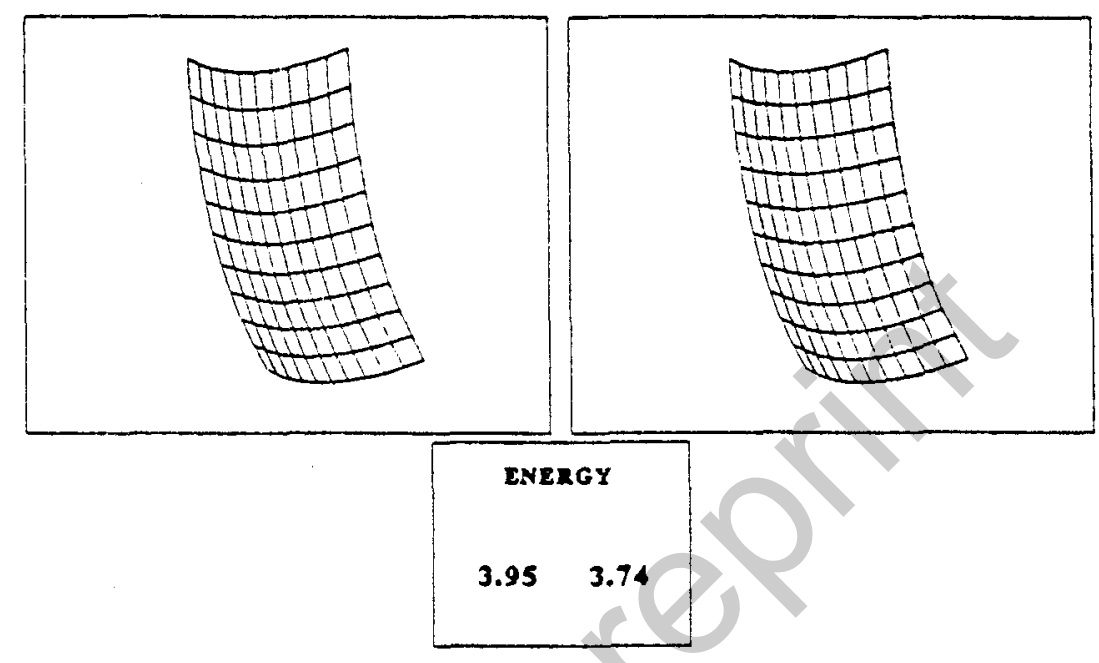

Figure 4:

The figures 5 and $6 *$ show the distribution of the bending energy on the same surface, with weights equal to one (figure 5 ) and with our optimal weights (figure 6 ).

\section{Surface Interrogation}

The geometric modeling of free-form curves and surfaces is of central importance for sophisticated CAD/ CAM systems. Apart from the pure construction of these curves and surfaces, the analysis of their quality is equally important in the design and manufacturing process. It is for example very important to test the convexity of a surface, to pinpoint inflection points, to visualize flat points and to visualize technical smoothness of surfaces. The purpose of this chapter is to introduce generalized focal surfaces as a new tool for surface interrogation. A critical survey on other surface interrogation methods is given in [HSG90]

Focal surfaces are special line congruences. Line congruences have been introduced in the field of visualization by Hagen and Pottmann [HPD91]. They can be used to visualize the pressure and heat distribution on an airplane, temperature, rainfall, ozone over the earth's surface, etc. However, the setting in this paper is different. Here, focal surfaces are used as a surface interrogation tool.

${ }^{*}$ See page C-542 for Figures 5 and 6. 
Given a set of unit vector $E(u, w)$ a so called line congrunence is defined by:

$$
L(u, w, z):=X(u, w)+z \cdot E(u, w)
$$

where $X(u, \mathrm{w})$ is the surface representation.

For $E(u, w)=N(u, w)$ ( $\mathrm{N}$ is the normal verctor surface) we speak of a normal congruence. The focal surfaces

$$
F_{i}(u, w):=X(u, w)+\kappa_{i}^{-1}(u, w) \cdot N(u, w) \quad i=1,2
$$

are special normal congruences, where $\kappa_{1}$ and $\kappa_{2}$ are the principle curvatures of the surface $X(u, \mathrm{w})$.

We now introduce a generalization of this "classical" focal surface concept to achieve a new surface interrogation tool.

The Generalized focal surfaces have the following form:

$$
F(u, w)=X(u, w)+a f\left(\kappa_{1}, \kappa_{2}\right), N(u, w) \text { with } a \in \mathbf{R}
$$

where the scalar function $f$ now depends on the the principal curvatures $\kappa_{1}=\kappa_{1}(u, w)$, $\kappa_{2}=\kappa_{2}(u, w)$ of $X$. The real number $a$ is used as a scale factor. If the curvatures are very small you need a very large number $\boldsymbol{a}$ to distinct the two surfaces $X(u, w)$ and $F(u, w)$ on the screen. Variation of this factor can also improve the visibility of several properties of the focal surface, for ex. one can get intersections clearer.

For different applications we use different functions $f\left(\kappa_{1}, \kappa_{2}\right)$ :

\subsection{Convexity test $f\left(\kappa_{1}, \kappa_{2}\right)=\kappa_{1} \cdot \kappa_{2}$}

For many applications it is neccessary to know whether a certain region is convex or not. The convexity of a surface can be described by its Gaussian curvature $K=\kappa_{1} \cdot \kappa_{2}$. A region is convex if and only if $K$ is positiv all over the region. The generalized focal surface can visualize the change of sign on the Gaussian curvature with the factor $f=\kappa_{1}, \kappa_{2} ; X(u, w)$ and $F(u, \mathrm{w})$ intersect at points of vanishing Gaussian curvature. (see figure 7 )*

\subsection{Visualization of flat points $f\left(\kappa_{1}, \kappa_{2}\right)=\kappa_{1}^{2}+\kappa_{2}^{2}$}

It is important to know whether a certain region contains flat points $\left(\kappa_{1}=\kappa_{2}=0\right)$. Flat points can cause problems in the manufactoring process and a convex surface with flat points looks to have dents. To test a surface for flat points or regions we use $f\left(\kappa_{1}, \kappa_{2}\right)=\kappa_{1}^{2}+\kappa_{2}^{2}$; a flat point exists, wherever both surfaces touch. (see figure 8 )*

\footnotetext{
${ }^{*}$ See page $C-543$ for Figures 7 and 8.
} 


\subsection{Visualization of the technical smoothness of a surface}

$$
f=\frac{\kappa_{1}^{2}+\kappa_{2}^{2}}{\kappa_{1}+\kappa_{2}}
$$

In the last couple of years, successful algorithms were developed to design and construct technically smooth curves and surfaces (see [HBS93]).

Technical smoothness means that the data can be immediately transfered to the milling process. In this production chain, one part is still missing, the visualization of technical smoothness as a quality test before the milling process. We close this gap with the next result.

The difference of the surface areas of the surface $X(u, w)$ and the generalized focal surface $F(u, w)$ in the sense of $f:=\frac{\kappa_{1}^{2}+\kappa_{2}^{2}}{\kappa_{1}+\kappa_{2}}$ is a measure for the technical smoothness of the surface $X(u, \mathrm{w})$.

This statement is based on the following theorem:

\section{Theorem :}

Supposed that $X(u, \mathrm{w})$ is a surface with $\kappa_{1} \neq-\kappa_{2}$

$$
F(u, w)=X(u, w)+a \frac{\kappa_{1}^{2}+\kappa_{2}^{2}}{\kappa_{1}+\kappa_{2}} N(u, w)
$$

If $X(u, w)$ is technically smooth in the sense of $\int_{s}\left(\kappa_{1}^{2}+\kappa_{2}^{2}\right) d s \rightarrow \min$. then

$$
|A(F)-A(X)| \rightarrow \min .
$$

$\boldsymbol{A}(\boldsymbol{F})$ and $\boldsymbol{A}(\boldsymbol{X})$ is the surface area of $F(u, \mathrm{w})$ and $X(u, w)$.

For a proof and more details see [HH92] Now at the end we give a focal analysis of an practical example. Industrial data of a part of a hair dryer are used. There are two composed surfaces consisting of several patches, (see figure $9 *$ at the bottom). For each surface you can see the generalized focal surfaces with $f=\kappa_{1}^{2}+\kappa_{2}^{2}$. This focal analysis shows the curvature behaviour of these surfaces.

\section{References}

[Far90] G. Farin. Curves and Surfaces for Computer Aided Geometric Design. Academic Press, second edition, 1990.

[For72] R. Forrest. Interactive interpolation and approximation by Bezier polynomals. Computer J., 15: 71 - 70, 1972.

[HB93] H. Hagen and G. P. Bonneau. Variational Design of Smooth Rational Bezier Surfaces. Computing Supplementum, 8: 133 - 138, 1993.

${ }^{*}$ See page C-543 for Figure 9. 
[HBS93] H. Hagen, G. Brunnett, and P. Santarelli. Variational Principles in Curve and Surface Design. Surv. Math. Ind., 3: 1 - 27, 1993.

[HH92] H. Hagen and St. Hahmann. Generalized Focal Surfaces: A new method for Surface Interrogation. In Proceedings, Visualization '96, Boston, 1992.

[HL89] H. Hoschek and D. Lasser. Grundlagen der geometrische Datenverarbeitung. Teubner Verlag, Stuttgart, 1989.

[HPD91] H. Hagen, H. Pottmann, and A. Divivier. Visualizing Functions on a Surface. The Journal of Visualization and Computer Animation, 2: 52 - 58, 1991.

[HS91] H. Hagen and P. Santarelli. Variational design of smooth b-spline surfaces. In H. Hagen, editor, Topics in Geometric Modelling. SIAM, 1991.

[HSG90] H. Hagen, Th. Schreiber, and E. Gschwind. Methods for Surface Interrogation. In Proceedings, Viszalization '90, pages 187 - 193, 1990. 